questions we are likely to ask in the next century...,' but because I was desperately trying to find something positive, something praiseworthy to say about it. One good thing I found is the Greek mythological story of the three sisters Klothos, Lachesis and Atropos who spin, apportion and cut the thread of life, respectively. There is more imagination, vision and suggestion in this 250-word myth mentioned in the opening pages of the book than in the remaining 233 pages.

In recent years, the market has been flooded with books on aging, covering the whole range from serious discussions of the theories and mechanisms of aging to self-help, quick-fix anti-aging miracles. Fortunately, this book does not belong to the latter category of books that give a bad name to the field of aging research. On the other hand, it does not contribute anything new to the field of biogerontology either. I could not find out to what 'End' this book is 'A Means'!

In his brief introduction, Clark compares the state of aging research with that of cancer research and expresses his hope for unified DNA-based answers to the complex phenomenon of aging. In the rest of the book, the author treads a welltrodden path of reviewing the whole field of biogerontology in 10 chapters. The book starts with a description of lifespan curves, maximum lifespans for selected species, and outdated correlations of body weight with maximum lifespan, before it goes on to a superficial discussion of the nature and evolution of senescence and death. What could have been an important discussion of the developmental genetics of senescence and lifespan, and the nature of senescence repressor and senescence effector gerontogenes is marred by an almost mystical presentation of 'death is the default state' kind of ideas. Clark briefly discusses premature aging syndromes, before he returns the reader's attention to Leonard Hayflick's discovery that somatic cells can undergo only a limited number of replications. It would have been instructive if the author had expanded on the important role of telomeres and telomerase. Even the chapter on the immunology of aging, which is supposedly the author's field of research, is superficial and uncritical. The remaining chapters are just general overviews of the research on the anti-aging and life-prolonging effects of calorie restriction, the role of free radicals and other oxidative stress in the occurrence and accumulation of macromolecular damage, and the aging of the brain. In the final chapter, 'A Conditional Benefit', William Clark makes an unsuccessful attempt to reduce aging and longevity to the genome, and to argue for life style modulations to achieve a healthy old age.

'A Means to an End' serves nobody's purpose. It neither quenches the public's thirst for scandal, miracle cures and false hopes, nor does it strike an intellectual chord with scientists. Indeed, the author is certainly not going to make either money or his name from the book. Although factually correct, the overall tone of the book is impersonal, uncritical and unexciting. The limited bibliography at the end does no justice to the vast literature available in the field of gerontology. In my view, the author of four previous books on immunology, sex and molecular medicine could and should have done much better in his fifth book on aging and death.

The author is at the Danish Centre for Molecular Gerontology at the University of Aarhus, Denmark.

\section{E-mail: rattan@imsb.au.dk}

DOI: $10.1093 / \mathrm{embo-reports/kvd043}$

\section{Brain history}

\section{Norman Saunders}

Mechanisms of Cortical Development. by David J. Price and David J. Willshaw Oxford University Press, Oxford, UK. 328 pages, $£ 69.50$

ISSN 0-19-262427-X

Single author, or as in this case, dual author textbooks are becoming rare in today's world of increasing specialization and explosion of factual knowledge. The big advantage of this type of book is that the reader gets a unified view of a field. This is difficult to achieve in multiauthored books, which even with the most diligent editors are likely to be a mosaic with gaps and style changes. However, the uniformity of single/dual authors may be at the cost of detail and accuracy when they stray from their areas of expertise.

David Price and David Willshaw join a select group of distinguished authors who have shown their breadth of expertise by providing specialist monographs on topics as diverse as 'Secretory Mechanisms of the Gastro-Intestinal Tract' and 'Human Baro-reflexes in Health and Disease'. Those interested in the development of the cerebral cortex should be grateful to the authors for providing a largely up-to-date book that seeks to explain what is known today about the fundamental mechanisms that underlie the development of the mammalian neocortex. The scope is broad indeed, covering topics at the cellular and molecular levels including: the early development of the telencephalon, its molecular regulation, axon guidance and the control of cortical connections. The general coverage of the monograph makes it useful not only to the non-expert who wants an entry into or update on the field, but the wide range of the book makes it also of benefit to experts, who want a view of related aspects in the context of their own work.

The introduction mentions future challenges, which helps to establish the scope of the volume. The authors make the case that understanding the mechanisms of cortical development will have a great impact on our ability to comprehend and eventually treat neurological disorders. They make the conscious (sic) decision, however, not to deal with the nature of consciousness on the grounds that it is not directly observable, but can only be inferred from behaviour-a view that may not be shared by cognitive neurobiologists. The one concession to this view of neurobiology is in the last chapter, which deals very briefly with face perception and language, both of which require consciousness.

Much of the authors' description of cortical development is based on what is known about the mouse. They reason that future use of molecular biology techniques and studies of mutant mice will substantially increase our understanding of human neurological disorders. As the authors demonstrate, this is already bearing fruit in understanding the earliest stages of neural tube development and the etiology of certain neurological disorders. But proper understanding of human conditions requires knowledge of development of the 
human neocortex; a short summary of the information available would have been helpful.

Consideration of a wider range of species would have helped other sections of the book as well. Results from Richard Mark's group in Canberra working on marsupials could contribute to the discussion on whether a period of 'waiting' of thalamocortical afferents in the subplate region precedes their growth into the compact immature cortical plate. Similarly, there is still some controversy about the fate of subplate neurons: do they die in all species or do they die in some and fade away in others? I am not sure that many developmental 'neocorticologists' would accept the statement that the neuroepithelium provides the only source of all neurons for the neocortex, whereas the subventricular zone just produces astrocytes and oligodendroglia.

One of the things a non-expert looks for in a general text is some guidance on the value of experimental evidence and pitfalls for the uninitiated to watch out for. This is well done in some sections, particularly in those dealing with molecular genetics. In others, a bit more background would have been helpful. For example the authors mention in several places that studies which identify nuclei with DNA breaks were the first evidence that in the early neocortex programmed cell death occurs in as many as $50-70 \%$ of neuroepithelial cells on some gestational days. Here, a consideration that breaks in DNA are an inevitable indication of apoptosis would have been useful. Is there evidence that in rapidly dividing tissues such as the neuroepithelium, such breaks inevitably lead to cell death? Several reviews published in 1999 are substantially more up to date on the general aspects of cell death (apoptosis) than is covered in chapter 6 . Particularly striking is the fact that the authors did not mention caspases in this context.

It is always a difficult task in a book of this scope to make appropriate choices about references and there is a lack of balance in some areas. Considerable prominence is given to the 'Oxford School' of visual cortex development, where a more even-handed account would have given more space to the later work of Hubel and Wiesel, and of others. The section on barrel formation gives little inkling of the intellectual dominance of Hendrik Van der Loos in this field. His idea of how the periphery determines development of the somatosensory cortex is in one of the references cited, but it is not clearly expressed in the text. Later papers that develop the idea further and provide compelling experimental evidence are not cited at all.
Two useful features of the monograph are the extensive bibliography, which would have been even more useful as a searchable $C D$, and a glossary of terms and abbreviations. The latter is a bit uneven and contains at least one unacceptable error: horseradish peroxidase is not 'an electrondense enzyme.' Its reaction product is electrondense.

Although this monograph involved a great deal of work for the authors in the preparation, its presentation carries with it sepia-tinted reminders of books from the 1950s. After more than 50 years and 48 publications, perhaps the Physiological Society should review its policy on monographs. In this one, almost all of the illustrations are line drawings, many inaccurate or unclear. Some sources are incorrect or missing. The Physiological Society has already done a good job of modernizing its Journal of Physiology. Perhaps the same effort should be put into their monographs. The authors' text deserves better visual support and a much wider audience would be reached.

The author is professor of physiology at the University of Tasmania.

E-mail: N.Saunders@utas.edu.au

DOI: 10.1093/embo-reports/kvd044 